\title{
Erratum to: Evaluation of a new device for measurement of hemoglobin A1c for Japanese subjects
}

\author{
Seiya Shimoda $\cdot$ Takako Maeda $\cdot$ Noboru Furukawa \\ Kenshi Ichinose $\cdot$ Kayo Taketa $\cdot$ Motoyuki Igata \\ Takafumi Senokuchi · Takeshi Matsumura $\cdot$ Eiichi Araki
}

Published online: 5 November 2013

(C) The Japan Diabetes Society 2013

Erratum to: Diabetol Int (2013) 4:112-116

DOI 10.1007/s13340-012-0102-y

In the original publication, there are errors in the 'Results' section of the 'Abstract'. The correct sentence should appear as given below:

There was a strong positive correlation between HbA1cHPLC $(Y)$ and HbA1c-new device $(X)(Y=0.952 X+$ $0.147, r=0.989 ; p<0.001 ; 95 \%$ confidence interval of the slope $0.929-0.976 ; 95 \%$ confidence interval of the intercept -0.036 to 0.329 ).

The online version of the original article can be found under doi:10.1007/s13340-012-0102-y.

S. Shimoda $\cdot$ T. Maeda $\cdot$ N. Furukawa $\cdot$ K. Ichinose $\cdot$ K. Taketa $\cdot$

M. Igata $\cdot$ T. Senokuchi $\cdot$ T. Matsumura .

E. Araki $(\square)$

Department of Metabolic Medicine, Faculty of Life Sciences,

Kumamoto University, 1-1-1 Honjo, Chuo-ku,

Kumamoto 860-8556, Japan

e-mail: earaki@gpo.kumamoto-u.ac.jp 